\title{
HUMAN HEALH PREVENTION BY DETECTION AND QUANTIFICATION OF TOXIC CHEMICAL COMPOUNDS
}

\author{
Venelin Terziev ${ }^{1}$ and Stoyanka Petkova - Georgieva ${ }^{2}$ \\ ${ }^{1}$ Full Member of the Russian Academy of Natural History, Professor, Eng., D.Sc. (National \\ Security), D.Sc. (Economics), D.Sc. (Social Activities), Ph.D., Russian Academy of Natural History, \\ Moscow, Russia, Vasil Levski National Military University, Veliko Tarnovo, Bulgaria University of \\ Rousse, Rousse, Bulgaria, terziev@skmat.com \\ ${ }^{2}$ Associate Professor, Ph.D., University „Prof. d-r Assen Zlatarov” - Bourgas, Bulgaria, \\ s.p.petkova@gmail.com
}

\begin{abstract}
There are various methods of detection and quantification of toxic chemical compounds (TCCs) and toxic industrial chemicals (TICs). The present research is on the most effective methods known for achieving a good human health prevention. The TCCs detection methods are described and analyzed by showing their advantages and disadvantages. Although incineration or other oxidative procedures are widely discussed methodologies for TCC detection and quantification, derive their widespread industrial use and environmental persistence from their resistance to oxidative degradation. The more highly chlorinated the TCC, the greater its resistance to oxidation, and the longer it will persist in the environment. Several detection methodologies for TCC remediation have been described, including treatment with Chemical Detector Paper, Colorimetric Detection Tubes, Infra-Red (IR) Spectroscopy, M21 Remote Sensing Chemical Agent Alarm, Travell RHC, etc.
\end{abstract}

Keywords: human health, detection, quantification, toxic chemical compounds, toxic industrial chemicals.

\section{INTRODUCTION}

The present research is done in order to help the easiest way of human health prevention from any kind of toxic chemical exposer to be detected by the proper method and device. The toxic chemical compounds (TCCs) and the toxic industrial chemicals (TICs) are present in substantial quantities in environmental sediments. Indeed, TCCs tend to attach to the surface of organic matter, clay, and micro-particles that are still suspended in the water or have already settled down. They can remain buried in sediments for a long time and be slowly released into the water and evaporate into air. Releases through water into air occur especially when it is warm and when TICs concentrations in sediment are high as this keeps the TICs levels in water elevated.

TCCs and TICs have been released to the environment solely by human activity but also vice versa they react harmfully to the human health. TCCs and TICs predominantly are redistributed from one environmental compartment to another (e.g., soil to water, water to air, air to water, sediments to water). The majority of TCCs in air result from volatilization of TICs from soil and water. Some TCCs may be released to the atmosphere from uncontrolled landfills and hazardous waste sites, incineration of TICs containing wastes, 
leakage from older electrical equipment in use, and improper disposal or spills. TCCs may be released to water from accidental spillage of TICs - containing hydraulic fluids, improper disposal, combined sewer overflows or storm water runoff, and from runoff and leachate from TCCs - contaminated sewage sludge applied to farmland. TICs may be released to soil from accidental leaks and spills, releases from contaminated soils in landfills and hazardous waste sites, deposition of vehicular emissions near roadway soil, and land application of sewage sludges containing TICs. TCCs are globally circulated and are present in all environmental media.

It is important to know how to detect all of the TCCs and TICs danger at the environment, so the human health to be prevented.

\section{HUMAN HEALH PREVENTION BY DETECTION AND QUANTIFICATION OF TOXIC CHEMICAL COMPOUNDS}

\section{- Chemical Detector Paper}

Detector paper (Figure 1) (Chemical Agent Detection) is one of the least sophisticated and least expensive techniques for CA detection, yet it provides a very sensitive way of detecting liquids and aerosols. Normally detection paper contains two dyes and a pH indicator which are integrated into the cellulose fibres of the paper. When a drop of CA is absorbed by the paper, it dissolves one of the dyes, producing a visible colour change in the paper (2005; Davis). The colour will change according to the type of agent present, for example HD dissolves the red dye, nerve agents dissolve the yellow dye and VX dissolves the yellow dye as it is a nerve agent but also causes the $\mathrm{pH}$ indicator to turn blue thus creating a green-black color change. Chemical detection paper has the disadvantage that it lacks specificity and can result in false positives as it is known to react with common chemicals such as brake fluid, antifreeze, and insect repellent. False readings are especially undesirable in civilian situations because they may lead to mass panic. Therefore, it is strongly advised that chemical detection paper always be used in conjunction with another detector to increase accuracy (2005; Davis). The detection papers currently in service by the US Military are M8, M9 and 3-way detection paper (Creaser et al. 2004).

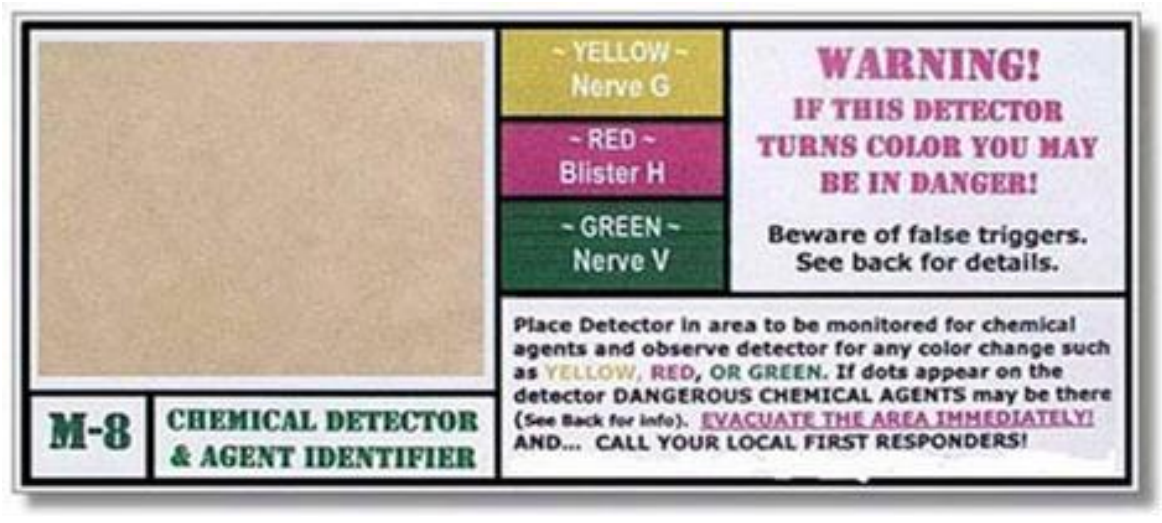

Figure 1. M8 Detection Paper.

M9 detection paper, shown in Figure 2 (Plackes, 2007), was developed for the U.S. military to enable soldiers to detect ' $G$ ', ' $V$ ' and ' $H$ ' agents in combat. It is still used by ground forces and is placed on personnel and equipment to enable detection of potential liquid CA aerosols (Chemical Agent Detection).

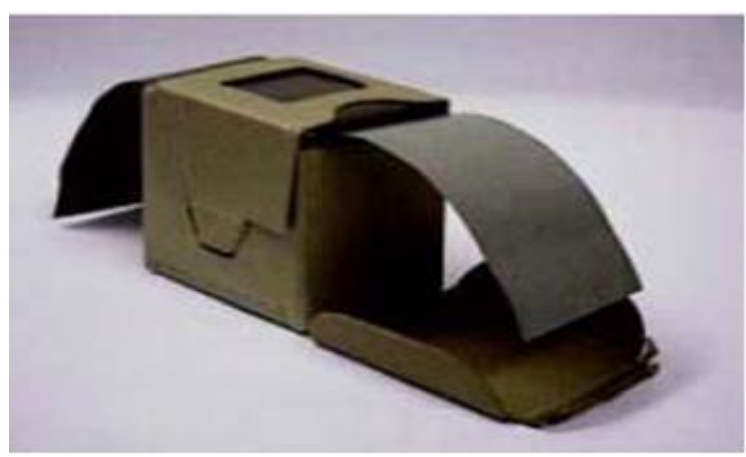

Figure 2. M9 Detection Paper. 


\section{- Colorimetric Detection Tubes}

Colorimetric tubes monitor one analyte per test tube and are used to detect gaseous or vaporous TCC. They have been utilised for years by HazMat teams as they are easy to use and familiar to the first responder community (2005; Creaser et al. 2004). The tubes generally consist of a glass tube containing a sorbent material which has had a reagent solution applied to it. When required for use, the tips of the tubes are broken off and a pump is used to draw a sample through it. If a CA is present the sorbent material will change colour. There are many different chemical reagents used in colorimetric tubes, since they are each specific to an agent or compound (2005; Coutant, 1999). Detection tubes are typically used for qualitative determinations to verify the presence of a CA after an alarm is received from another monitor (2005). A typical tube detection system consists of four or five tubes connected to a small pump which pulls a vapour or gas sample through all the tubes at a constant rate. A response is usually achieved within a few minutes however the results are heavily dependent on the analyte being tested as well as the concentration and the flow rate of sample through the tube (Creaser et al. 2004). Colorimetric tubes, such as those available from Dräger (Lübeck, Germany), shown in Figure 3, are used extensively, however there are some disadvantages. At present there are 160 substance-specific reagent tubes available for identifying different agents. Hence to utilise this technique effectively prior knowledge of which CA is likely to be present is required otherwise a tube for each possible CA must be used for thorough detection and to avoid false negatives (Davis).

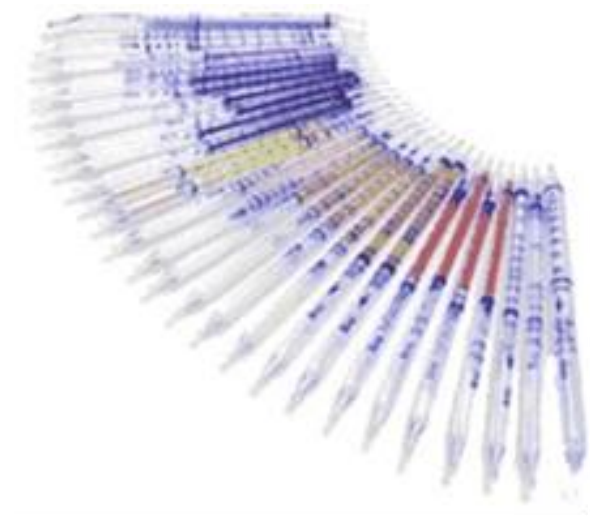

Figure 3. Drager Colorimetric Tubes.

- Infra-Red (IR) Spectroscopy

IR is employed in several point and standoff TCC detectors. For field applications IR-based detectors are used to determine whether a sample contains targeted chemicals rather than being used to identify them (Sun, Ong, 2005; Davis). In IR spectroscopy, IR radiation is passed through a sample and some of this radiation is absorbed whilst some is transmitted. The result is the production of a spectrum which represents the molecular absorption or transmission, creating a unique molecular fingerprint of the sample (Introduction to Fourier Transform Infrared Spectrometry).

IR instruments measure the amount of light absorbed at a specific wavelength to look for a characteristic chemical group, such as the phosphorus-oxygen bond of nerve agents (Sun, Ong, 2005, Creaser et al. 2004). The intensity of this IR absorption is proportional to the concentration of the targeted chemical10. The IR region of the electromagnetic spectrum ranges from 0.78 to 1000 microns $(\mu \mathrm{m})$ (Creaser et al. 2004) and can be further subdivided into the near, mid and far IR regions which range from $0.78 \mu \mathrm{m}$ to $2.5 \mu \mathrm{m}$ (or wave numbers $12800-4000 \mathrm{~cm}-1), 2.5 \mu \mathrm{m}$ to $50 \mu \mathrm{m}(4000-200 \mathrm{~cm}-1)$ and $50 \mu \mathrm{m}$ to $1000 \mu \mathrm{m}(200-10 \mathrm{~cm}-1)$, respectively. The most common wavelengths for detection applications occur in the mid IR region and range between $2.5 \mathrm{~m}$ and $15 \mu \mathrm{m}(4000-670 \mathrm{~cm}-1)$ (Sun, Ong, 2005; 1999). The characteristic wavelengths of $\mathrm{GA}, \mathrm{GB}$ and $\mathrm{HD}$ have been determined to be $9.7,9.9$ and $13.9 \mu \mathrm{m}$, respectively (Longworth, Barnhouse, Ong, 2000).

\section{- IR Technology}

A sample is drawn into, or continuously through, a sample cell of fixed dimensions. IR radiation enters the sample cell through an IR-transparent window which is set perpendicular to the radiation path. The window and sample cell are orientated to permit maximum IR radiation to pass through the cell. The radiation can reach the absorption photometer in either a single pass, or in multiple passes via the use of properly aligned mirrors which reflect the IR beam back and forth through the sample multiple times. This can increase the effective path length to a maximum, which subsequently results in the highest sensitivity being achieved 
(Sun, Ong, 2005). IR absorption is then detected by either an IR transducer or via a photoacoustic method. The most common transducers include thermal, piezoelectric and photo- conducting transducers. They act by changing the received IR signal to an electrical signal which can then be processed. Photoacoustic methods, however transform electromagnetic radiation into acoustic waves (Sun, Ong, 2005). At present there are several different detection techniques that utilise IR spectroscopy. They include photoacoustic IR spectroscopy, filter-based IR spectroscopy, passive IR detection, including forward-looking IR spectroscopy (FLIR), and Fourier Transform IR spectroscopy (FTIR) (Davis).

\section{- Advantages}

IR based detectors have the advantage of having reasonably high sensitivity, low LOD and fast detection of vapours. Furthermore, IR is a non-destructive technique which can handle a large sample volume whilst requiring minimal, if any, sample preparation prior to analysis (Sun, Ong, 2005).

A sample is introduced into the sample cell, which is closed during the analysis enabling the sample to be subjected to a number of different wavelengths of IR radiation. Due to IR not affecting the integrity of the sample, it is highly recommended that the sample be checked using one or more additional characteristic wavelengths if the detected sample produces an alarm at the initial wavelength. This is largely due to the fact that various chemicals may contain functional groups that absorb IR at similar wavelengths, and as a result detection determination based on single wavelengths may result in higher false positive and/or negative alarm rates due to reduced discriminatory ability (Sun, Ong, 2005). FTIR has the added advantage of providing a precise measurement method requiring no external calibration. It can increase speed and sensitivity as scans can be collected every second and then can be added together to ratio out the random noise. More importantly FTIR based detectors are mechanically simple as they only contain one moving part, hence there is very little possibility of mechanical breakdown (Introduction to Fourier Transform Infrared Spectrometry).

\section{- Disadvantages}

Major limitations associated with IR-based detectors are cost, complexity and size of instrumentation (Creaser et al. 2004). Environmental conditions may also significantly affect an IR-based detector's performance, for example, relative humidity and changes in sample moisture level may generate false positive absorption responses and interferences which may mask peaks of interest. This effect can be reduced by utilising a filter to measure background moisture content (Sun, Ong, 2005).

Currently available IR spectrometers offer a limited level of standoff detection, whilst photoacoustic-based IR techniques are very sensitive to vibration and environmental effects and as such their use in handheld detection devices for field operations is limited (Sun, Ong, 2005, Creaser et al. 2004).

Furthermore, tested detectors based on IR techniques do not have sufficient sensitivity to detect TCC vapours at IDLH levels. However, given the ability of these instruments to reliably identify targeted chemicals, they could become useful tools in assessing incidents where the concentration is above IDLH levels (Sun, Ong, 2005).

\section{- Existing IR- based Detectors}

Detection devices using IR techniques in field applications include remote and point sample detectors. Remote devices that detect IR radiation changes in the background include the M21 detector and the Joint Service Lightweight Standoff Chemical Agent Detector (JSLSCAD), both of which are used by the US military in field operations (Sun, Ong, 2005). The MIRAN SapphIRE Portable Ambient Air Analyser is a portable filter- based IR instrument, whilst the AN/KAS-1 and AN/KAS-1A Chemical Warfare Directional Detectors are FLIR based. The TravellR HCl, HazMat ID and IlluminatIR all employ IR spectroscopy.

- M21 Remote Sensing Chemical Agent Alarm

The M21 Remote Sensing Chemical Agent Alarm (RSCAAL), shown in Figure 4 was manufactured by Intellitec (Deland, Florida) and was the first fielded standoff chemical detection device based on passive IR detection (Davis). 


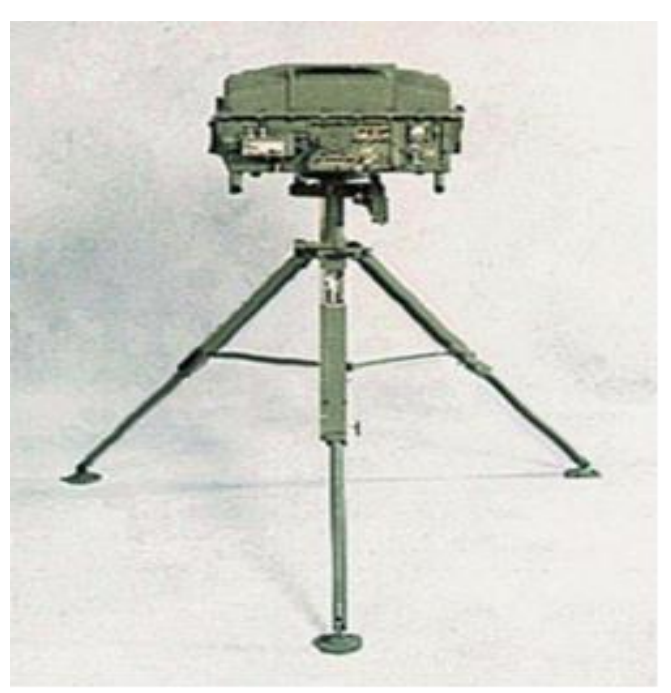

Figure 4: M21 RSCAAL.

This detector operates in the 8-12 $\mu \mathrm{m}$ region of the IR spectrum and has an interferometer that collects absorption or emission spectra from a TCC cloud and compares it to a previously collected background spectra. As a result the M21 RSCAAL is able to detect nerve and blister agent vapour clouds at line of sight distances out to $5 \mathrm{~km}$ and along a $60^{\circ}$ arc, in seven fieldof-view segments (Davis). Detection is therefore based on changes in the IR energy emitted from remote objects, or from clouds formed by an agent. This became a desired detector due to its ability to automatically scan the surrounding environment and subsequently give early warnings of an attack thus allowing commanders to identify and manoeuvre around contaminated areas.

When the M21 RSCAAL detects a CA, the alarm light illuminates and the horn sounds. Additionally, small field-of-view lights will illuminate to inform the operator in which fieldof-view the agent was detected. If the agent cloud has been tracked whilst moving, it is possible that all field-of-view lights will be illuminated.

The M21 is two-man portable and can be set up in approximately 10 minutes. It is generally unaffected by low light conditions, however it is limited in that it must be stationary and it can be obstructed by snow, rain and dust clouds (Davis). No independent evaluation has been conducted to assess the effectiveness of this detector.

\section{- TravellR HCl}

In 2001, SensIR Technologies (Danbury, CT) introduced the TravellR, shown in Figure 5 which was claimed to be the first portable FTIR spectrometer that could rapidly identify an unknown substance in situations where there was clearly a visible threat. The TravellR $\mathrm{HCl}$ is referred to as an identifier not a detector as it is not fitted with a gas cell for vapour detection and is normally used in conjunction with other traditional detection equipment48. Since its introduction, it has been widely used for the identification of a range of unknown materials including TCC, explosives, narcotics and other common chemicals. For normal operation, the TravellR HCl must firstly be connected to a laptop. The sample is then placed on top of a diamond crystal embedded in a stainless steel disk, called a DuraDisk. The IR beam passes through the crystal, which has a high refractive index, and penetrates the sample producing an IR spectrum which is then compared to an IR spectra of reference compounds in the database library. This process is non- destructive.

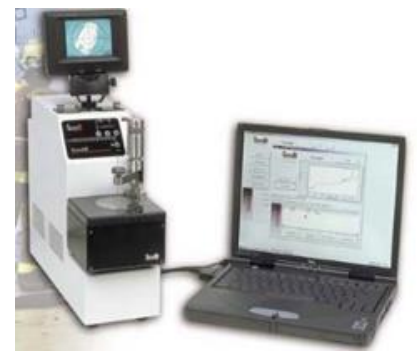

Figure 5. TravellR $\mathrm{HCl}$.

There are three variations of the stainless steel DuraDisk available: (i) the Three Reflection disk which provides flexible analysis on solid or liquid samples, (ii) the Single Reflection Diamond Attenuated Total 
Reflection (ATR) sample disk, shown in Figure 6 which is used for solid samples and (iii) the Nine Reflection disk, also shown in Figure 6, which is used for liquid samples only. The Volatile Cover, as the name implies, is a clear plastic cover that is placed on top of the sample to minimise sample loss during analysis due to high volatility.

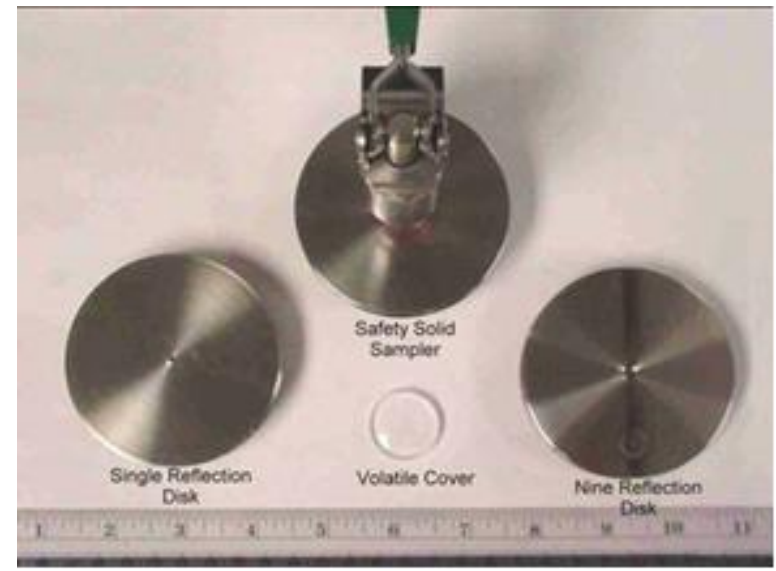

Figure 6: TravelIR $\mathrm{HCl}$ sampling accessories.

\section{CONCLUSION}

The use of TCCs has been restricted for many years and banned for use in new kind of goods that could be exposed in the environment. Nevertheless, large numbers of TICs-contaminated transformers are still in service. The detection of TCCs downstream confirmed and give an indication of the migration pattern of TCCs which are very stable and hence their reason for their persistence (Terziev, Petkova - Georgieva, 2019-a). Although, production of TICs halted 25 years ago, low levels are still found in air, water, sediment, soil and food. Levels are declining. Urban areas have higher levels. Indoor levels may be higher than outdoors. Despite low solubility, TICs are widespread in water bodies. Typical levels of TICs in the ocean and the Great Lakes are 0.02 to $0.6 \mathrm{ng} / \mathrm{L}$. Storm water runoff and rainwater are commonly contaminated with TICs. Rainwater concentrations from 1980-1990 were in the range of 0.1 to $20 \mathrm{ng} / \mathrm{L}$. Declining but widespread, low-level TICs contamination is present throughout the environment. Higher levels of TICs contamination are associated with many former manufacturing and industrial sites. Since TCCs are mixtures they are expected to have toxic interactions with other chemicals. Indeed, TCCs are routinely used to increase the sensitivity of a variety of bioassays by inducing hepatic enzymes which alter the metabolism of other chemicals and hence alter their toxicity (Petkova - Georgieva, 2018).

\section{REFERENCE LIST}

Chemical Agent Detection. In Security and Safety Supplies.

Guide for the Selection of Chemical Agent and Toxic Industrial Material Detection Equipment for Emergency First Responders, (2005). Guide 100-04, Volume I and II: Summary; SAVER July, 2005; pp 1-5.

Davis, G. CBRNE - Chemical Detection Equipment.

Creaser, C. S., Griffiths, J. R., Bramwell, C. J., Noreen, S., Hill, C. A., Thomas, C. L. P. (2004) Ion Mobility Spectrometry: a review. Part 1 . Structural analysis by mobility measurement. The Analyst 2004, 129, 984- 994.

Plackes, J. (2007). NBCDE Detection Graphics. 2007.

Coutant, S.(1999). Features, Chemical Warfare Agent Detection and Personal Protection. Jane's Defence Weekly 1999.

Sun, Y., Ong, K. Y. (2005). Detection technologies for Chemical Warfare Agents and Toxic Vapors. 1st ed.; CRC Press: Boca Raton, Florida, 2005; p 272.

Introduction to Fourier Transform Infrared Spectrometry. In Nicolet, T., Ed. Thermo Nicolet: Madison, pp 1-7. Committee on R\&D Needs for Improving Civilian Medical Response to Chemical and Biological Terrorism 
Incidents (1999), I. o. M., Detection and Measurement of Chemical Agents. In Chemical and Biological Terrorism: Research and Development to Improve Civilian Medical Response, National academy press: Washington, D.C., 1999; pp 43-64.

Longworth, T. L., Barnhouse, J. L., Ong, K. Y. (2000). Domestic Preparedness Program: Testing of MIRAN SapphIRe Portable Ambient Air Analyzers Against Chemical Warfare Agents Summary Report; Aberdeen Proving Ground, Marylands, July, 2000; pp 1-13.

Petkova - Georgieva, St. (2018). Basic Problems to Solve in Strategic Controlling Implementation, annual of "Prof. Dr. Assen Zlatarov", pp. 71 - 77, v. XLVII, book 2, Burgas, Bulgaria, 2018, ISSN 2603-3976.

Terziev, V., Petkova - Georgieva, S. (2019). The performance measurment system key indicators and the determinants impact on the level of decentralization using as an example a subdivisional unit from the Bulgarian social health and care experience. // Proceedings of SOCIOINT 2019-6th International Conference on Education, Social Sciences and Humanities 24-26 June 2019- Istanbul, Turkey, International Organization Center of Academic Research, Istanbul, Turkey, 2019, pp. 515-524, ISBN: 978-605-82433-6-1.

Terziev, V., Petkova - Georgieva, S. (2019a). A research study of nonlinearity experiencing in the rate of current account deficit to the Bulgarian health and care national product. // Proceedings of SOCIOINT 2019- 6th International Conference on Education, Social Sciences and Humanities 24-26 June 2019Istanbul, Turkey, International Organization Center of Academic Research, Istanbul, Turkey, 2019, pp. 525-533, ISBN: 978-605-82433-6-1. 